\title{
Prevalencia y patrones de consumo de alcohol en adolescentes de secundaria en una comunidad rural
}

De la Cruz-Irineo Elizabeth*, Reséndiz-Avendaño Carmen Lorena*, Chávez-González Laura Lucero*, Hernández-Castañón Ma. Alejandra**, Garza-González Beatriz*, Zamora Mendoza Aurora**

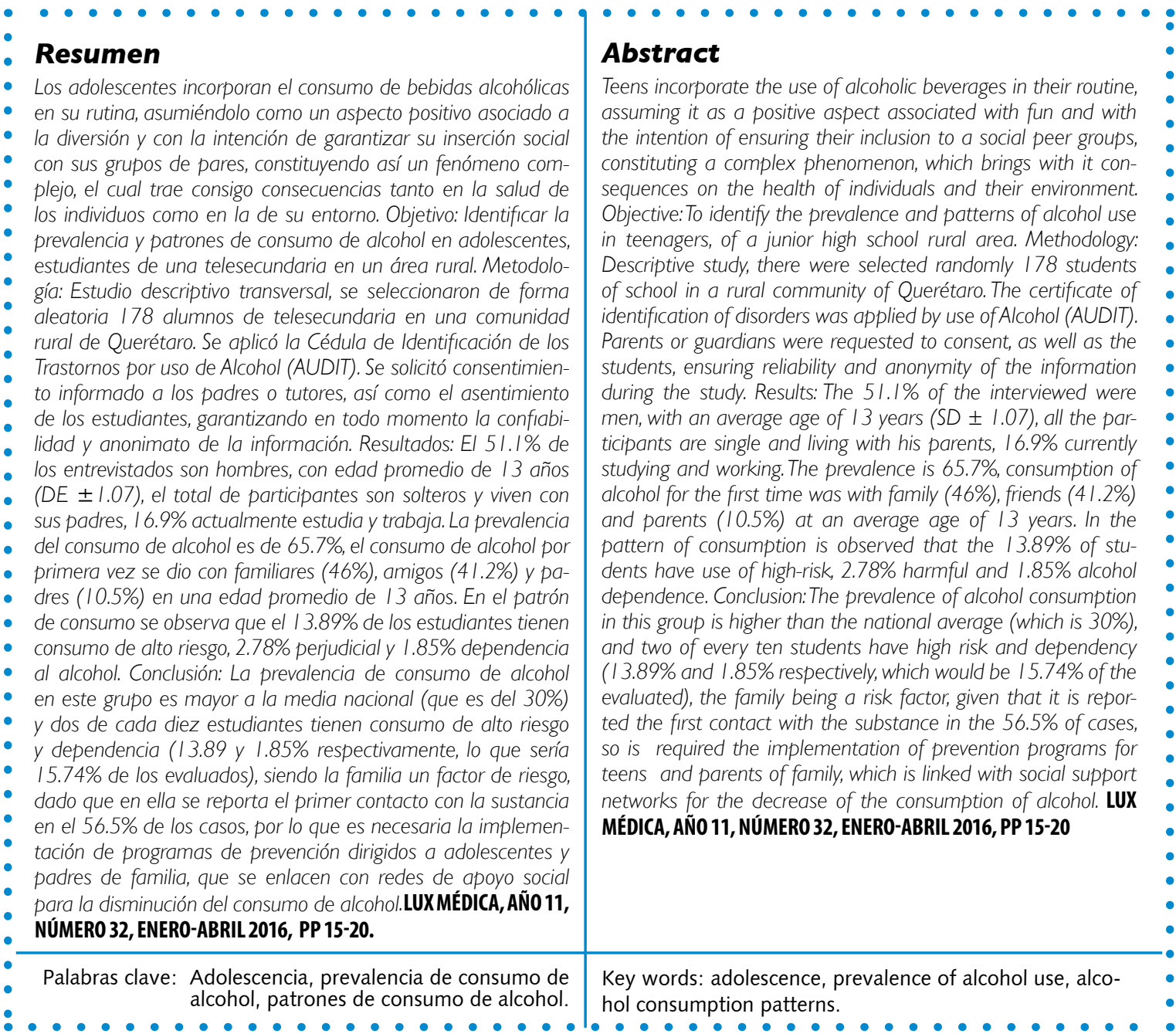

* Pasantes en Servicio Social. Licenciatura en Enfermería, Facultad de Enfermería. Universidad Autónoma de Querétaro

** Docentes- Investigadoras del CA-4: Salud y Educación. Universidad Autónoma de Querétaro.

Fecha de recibido: 29 de septiembre de 2015

Fecha de aceptación: 9 de febrero de 2016

Correspondencia: Ma. Alejandra Hernández Castañón. Facultad de Enfermería. Universidad Autónoma de Querétaro. Cerro de las Campanas S/N Las Campanas 76010 Santiago de Querétaro, México. Teléfono 01442192 1200. Correo electrónico alehdez983@yahoo.com.mx 


\section{Introducción}

La adolescencia es una etapa de la vida marcada por intensas transformaciones físicas, emocionales e influencias socioculturales que perduran desde la pubertad hasta la vida adulta, caracterizada principalmente por la búsqueda de nuevas experiencias y respuesta a interrogantes ${ }^{1}$.En este sentido, los adolescentes incorporan el consumo de bebidas alcohólicas en su rutina con el propósito de garantizar su inserción social en grupos de jóvenes que validan aspectos positivos del consumo de alcohol y minimizan las consecuencias en la salud individual y de su entorno ${ }^{1}$.

En este orden de ideas, Ferreira, Méndez y Teixeira ${ }^{2}$, refieren que el alcohol es una sustancia psicoactiva capaz de afectar el sistema nervioso central, con efectos tales como la sensación de relajamiento, desinhibición e incluso euforia, puede comprometer el discernimiento y ocasionar periodos de pérdida de memoria. Este proceso puede llevar a la dependencia, por lo cual se incorpora cada vez más a la vida cotidiana de la persona y en específico de los adolescentes, debido a efectos sociales (como la integración a un grupo en específico) y psicológicos (como el alivio a algún malestar personal o situacional).

Ante esta situación, se considera que el consumo de alcohol es el primer factor de riesgo sanitario en los países desarrollados al afectar al individuo en los niveles biológico, físico y psicológico, además de relacionarse con accidentes automovilísticos, violencia, suicidio, incluso como un impacto directo en la carga sanitaria de los países ${ }^{3}$.

En México, la ingesta de bebidas alcohólicas es cada vez más aceptada por la sociedad por el valor cultural que se le otorga, al ser parte importante en festividades religiosas y familiares, costumbre que aumenta con frecuencia en poblaciones con características demográficas rurales, es decir, localidades con menos de 2500 habitantes $^{2}$.

En este sentido, la Secretaría de Salud ${ }^{4}$ indica una alta variabilidad (entre 7\% y 40\%) de consumo de alcohol en jóvenes de 15 a 18 años; lo anterior responde a la falta de consenso a la hora de definir el Consumo Intensivo de Alcohol (CIA) y a las diferencias de etanol puro que contiene una Unidad de Bebida Estándar (UBE) en cada país. Así, en los estudios se han empleado distintos puntos de corte en cuanto al número de UBEs consumidas por ocasión en los diferentes intervalos de frecuencia en los que se producen los episodios de consumo intensivo ${ }^{5}$.

Por otro lado, en el estado de Querétaro "el consumo de bebidas alcohólicas en el sector joven de la población se caracteriza por una mayor cantidad de ingesta en un solo evento, el $25.2 \%$ de los jóvenes consumen hasta dos bebidas, mientras $38.1 \%$ beben hasta cuatro, el $17.4 \%$ hasta seis y el $16.7 \%$ más de siete bebidas, por lo que el consumo de bebidas alcohólicas se ha incrementado cerca de cinco puntos porcentuales" 6 . El objetivo de este trabajo fue identificar la prevalencia y patrones de consumo de alcohol en adolescentes de una comunidad rural en el estado de Querétaro. 


\section{Material y Método}

Se realizó un estudio prospectivo, descriptivo y transversal en estudiantes de telesecundaria de una comunidad rural de Querétaro. Se incluyeron 178 adolescentes de ambos sexos, con edades entre 11 y 16 años, inscritos en la institución educativa en estudio. Se elaboró una base de datos para procesar la información obtenida, utilizando el Paquete Estadístico Informático SPSS V.20 (Statistical Package for the Social Science). En el estudio se garantizó la confidencialidad y anonimato de la información, de igual forma se contó con el consentimiento informado de los padres o tutores y el asentimiento de los estudiantes participantes en el estudio y de las autoridades de la institución.

\section{| | | | | | | | | | | | | | | | | | | | | | | | | | | | | | | | | | | | | | | | | | | | | | | | | | | | | | | | | | | | | | | | | | | | | | | | | | | | | | | | | | | | | | | | | | | | | | | | | | | | | | | | | | |}

\section{Resultados}

De un total de 178 estudiantes de telesecundaria de una comunidad de Querétaro, el $51 \%$ son hombres, el total de la muestra manifiesta vivir con sus padres. Se distribuyeron por grado escolar de la siguiente forma: tercer grado $(27 \%)$, segundo grado $(38 \%)$ y primer grado (35\%) (tabla 1). La edad promedio fue de 13 años (DE \pm 1.07 ) y un promedio escolar reportado por los estudiantes de 8.9 ( $\mathrm{DE} \pm 1.43$ ).
En cuanto a la prevalencia del consumo de alcohol, se identifica que el $65.7 \%$ de los encuestados ha consumido alguna vez en su vida, con una edad de inicio promedio de 13 años, sin embargo, es importante señalar que un $23 \%$ refiere su edad de inicio entre los 5 y 11 años, observándose como factor de riesgo el núcleo familiar, ya que el $56.5 \%$ inició este consumo con sus padres o familiares. El $44.4 \%$ reportó consumo en el último año y el $55.6 \%$ en el último mes (tabla 2).

\section{Tabla I}

\section{Características socio-demográficas de participantes en el estudio}

\begin{tabular}{|llcc|}
\hline & Variable & F & \% \\
\hline Sexo & Mujer & 87 & 48.9 \\
\hline & Hombre & 91 & 51.1 \\
\hline Edad & De 11 a 13 & 107 & 60 \\
\hline & De 14 a 16 & 71 & 40 \\
\hline Estado Civil & Soltero & 178 & 100 \\
\hline Vive con & Padres & 178 & 100 \\
\hline Trabaja & Sí & 30 & 16.9 \\
\hline & No & 148 & 83.1 \\
\hline Grado & $1^{\circ}$ & 65 & 36.5 \\
\hline & $2^{\circ}$ & 66 & 37.1 \\
\hline $3^{\circ}$ & 47 & 26.4 \\
\hline
\end{tabular}

Fuente: Cédula de Identificación. 


\section{Tabla 2}

\section{Prevalencia del consumo de alcohol}

\begin{tabular}{|c|c|c|c|}
\hline \multirow{2}{*}{$\begin{array}{l}\text { Variable } \\
\text { Consumo alcohol }\end{array}$} & & $\mathbf{F}$ & $\%$ \\
\hline & Sí & 117 & 65.7 \\
\hline No & 61 & 34.3 & \\
\hline Consumo de alcohol & En el último año & 52 & 44.4 \\
\hline En el último mes & 65 & 55.6 & \\
\hline A qué edad probaste alcohol & Menos de 5 años & 5 & 3.0 \\
\hline De 5 a 8 años & 7 & 4.0 & \\
\hline De 8 a 11 años & 28 & 16.0 & \\
\hline De 11 a 14 años & 70 & 39.0 & \\
\hline De 14 a 16 años & 8 & 4.0 & \\
\hline Consume alcohol con: & Solo & 6 & 5.3 \\
\hline Amigos & 47 & 41.2 & \\
\hline Padres & 12 & 10.5 & \\
\hline Familiares & 49 & 46.0 & \\
\hline Consumo de alcohol & En el último año & 52 & 44.4 \\
\hline En el último mes & 65 & 55.6 & \\
\hline
\end{tabular}

Fuente: Elaborado a partir del AUDIT

En relación con los patrones de consumo, se muestra que el $81.48 \%$ tiene un consumo abstemio y de bajo riesgo, sin embargo, es importante resaltar que el
$13.89 \%$ tiene un consumo de alto riesgo, $2.78 \%$ consumo perjudicial y $1.85 \%$ dependencia al alcohol (Figura 1).

\section{Figura I}

\section{Patrones de consumo de los participantes en estudio.}

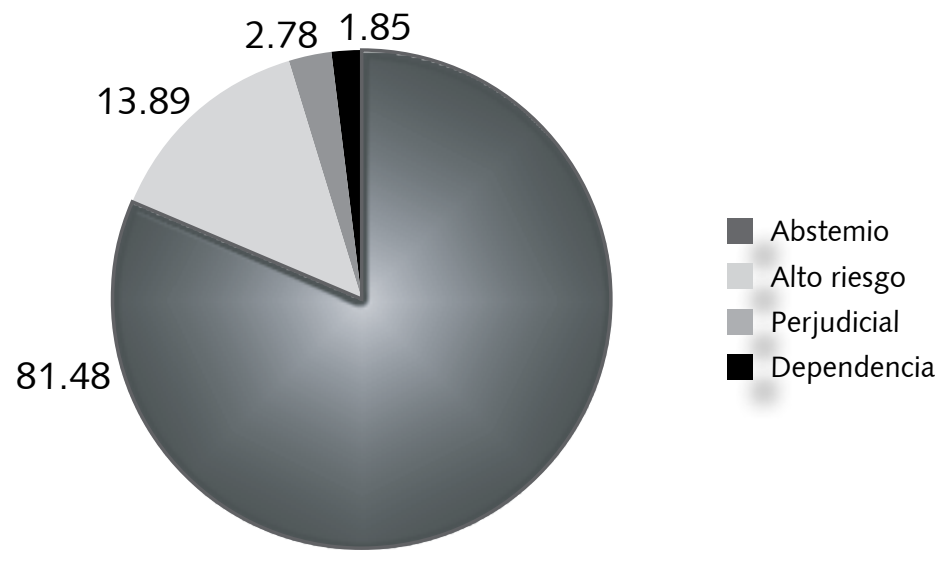

Fuente: A partir del AUDIT. 


\section{Discusión}

En relación con las características sociodemográficas de los adolescentes participantes en el estudio, éstas son similares a lo reportado por Álvarez, Zamora, Hernández, Sánchez, Barrón, Mendoza ${ }^{7}$ en el cual predominó el género masculino (51.4\%), edad entre 11 y 12 años (57.4\%), la mayoría vivían con sus padres (85\%) y menos del $20 \%$ estudia y trabaja.

La edad promedio de inicio de consumo de alcohol es de 13 años y se identificó a la familia y amigos como factor de riesgo, al referir los estudiantes que el primer contacto con el alcohol fue en reuniones con familiares y amigos, situación también referida por Valencia, García, Lozano ${ }^{8}$ en tanto que en otros estudios se menciona sólo a la familia. ${ }^{9}$

Por otra parte, el consumo abusivo de alcohol se relaciona positivamente con el consumo de la familia y los amigos $(\beta=$ $0.805, p<0.001)$ y se presenta más entre los hombres que entre las mujeres $y$, sobre todo, en varones jóvenes mayores de 15 años ${ }^{5}$, situación que también se presentó en el presente estudio, pues en una población menor de 16 años, el 13.89\% presentó consumo de alto riesgo y el $1.85 \%$ dependencia al consumo de alcohol, porcentajes que también son similares a lo reportado en la Encuesta Nacional de Adicciones (ENA 2011) ${ }^{10}$, con un $14.5 \%$ y $4.1 \%$ respectivamente.

\section{Conclusiones}

En la población rural evaluada, se identificó que la prevalencia del consumo de alcohol en los adolescentes es alta. Se resalta que dos de cada diez estudiantes ya tienen patrones de consumo abusivo y de adicción, por lo cual son de suma importancia actividades intersectoriales para coadyuvar en la generación de programas y espacios para actividades recreativas, con la participación de la comunidad para hacer frente a esta problemática social y de salud, donde enfermería y otras disciplinas deben asumir un papel de liderazgo en actividades comunitarias. La salud escolar también se convierte en un espacio importante para la intervención educativa a través de programas de prevención a las adicciones. Es necesario recuperar programas sobre adicciones que sean coordinados por las instituciones de salud e incluyendo a la comunidad y a la familia, para llevar a cabo acciones tendientes a la prevención y disminución del daño en la población adolescente, conformando redes de apoyo social en la comunidad. Estas acciones deben estar orientadas tanto a prevenir que los adolescentes inicien el consumo del alcohol, como a brindarles alternativas de estilos de vida saludable a quienes ya lo consumen.

\section{Bibliografía}

1 Krauskopf D. El desarrollo en la adolescencia: las transformaciones psicosociales y los derechos en una época de cambio. Rev Psiquiatria.com [Internet] 2011 [Citado el 10 de marzo de 2012]; 5(15) Disponible en: https://scholar.google.com.mx/scholar?clu ster $=1137707080728340417 \& \mathrm{hl}=$ es \&as $s \mathrm{dt}=0,5$.

2 Mendes L, Teixeira ML, Ferreira M. Alcohol in adolescence: the care-education as an action strategy for the nursing. Rev. Enferm Esc- Anna Nery [Internet] 2010 [Citado el 10 de enero de 2014]; 14(1) 158164. Disponible en: http://www.scielo.br/scielo. php?pid=S1414-81452010000100023\&script $=$ sci arttext. 
3 Elzo BR, Fernández AR. El consumo de alcohol en adolescentes escolarizados: propuesta de un modelo socio comunitario. Rev. Latino-Am. Enfermagem, [Internet] 2011 [Citado el 15 de marzo de 2012]; Disponible en:[HTML]El consumo de alcohol en adolescentes escolarizados: propuesta de un modelo socio comunitario. [HTML] de isciii.es.

4 Secretaria S. Programa contra el Alcoholismo y el abuso de bebidas Alcohólicas: Actualización 2011. 2012.1 $1^{\mathrm{a}}$ ed. México: Printed and made in México. 2011 [Citado el 15 de septiembre de 2013]. Disponible en:http://www.conadic.salud.gob.mx/pdfs/ publicaciones/abuso_de_bebidas.pdf.

5 Cicua $D$, Méndez M, Muñoz L. Factores en el consumo de alcohol en adolescentes. Rev Pensamiento Psicológico [Internet]. 2008 [Citado el 6 de julio de 2013]; 4(11) 115-134. Disponible en: http://revistas.javerianacali.edu.co/javevirtualoj/index.php/ pensamientopsicologico/article/view/83/247.

6 Rodríguez JL, Valderrama R, Ayala $M$, Martínez FJ. El consumo de alcohol, tabaco, drogas y comportamiento sexual en estudiantes de secundaria. Rev psicología Integrativa-BUAP. [Internet]. 2013 [Citado el 2 de enero de 2014]; 1 (1). Disponible en: http:// www.psicologia.buap. $\mathrm{mx} /$ revista/1/contraportada. pdf.
7 Álvarez $A$, Zamora $A$, Hernández $M A$, Sánchez $M$, Barrón $M$, Mendoza MA. Resiliencia y consumo de alcohol y tabaco en estudiantes adolescentes de comunidades rurales. Anuario de investigación en adicciones [Internet]2011 [Citado el 6 de diciembre de 2013]; 12(1) 15-21. Disponible en: http://www. cucs.udg.mx/avisos/anuario_investigacion_adicciones/Anuarios_PDFs/Anuario12.pdf\#page $=16$.

8 Valencia MJ, García MLR, Lozano MM. Consumo de alcohol en estudiantes de educación secundaria, aspectos personales y sociales relacionados. Rev Psicología. com [Internet]. 2011 [Citado el 20 de julio de 2013]; 15 (9). Disponible en: http://hdl.handle. net/10401/3449.

9 González EV, Sosa CS, Ochoa GM. Consumo abusivo de alcohol en adolescentes escolarizados: propuesta de un modelo psicocomunitario. Rev CIENCIAUANL [Internet] 2011[Citado el 15 de septiembre de 2013]; 15(4) 445-458. Disponible en: http://dialnet. unirioja.es/descarga/articulo/3814234.pdf.

10 Instituto NPRFM, Secretaría S. Encuesta Nacional de Adicciones 2011: Reporte de Alcohol. $1^{a}$ ed. México. Printed in México. 2012 [Citado el 24 de agosto de 2013]. Disponible en: www.inprf.gob.mx, www. conadic.gob.mx, www.cenadic.salud.gob.mx, www. insp.mx. 